Bayero Journal of Pure and Applied Sciences: 10(1): 634 - 641

ISSN 2006 - 6996

\title{
MODELLING THE DYNAMICS OF CHIKUNGUNYA IN HUMAN AND MOSQUITO POPULATIONS
}

\author{
Sabiu, S.M. ${ }^{a, b}$ and Hussaini, N. ${ }^{a, 1}$ \\ A Department of Mathematical Sciences, Bayero University Kano, P.M.B.3011, Kano, Nigeria.

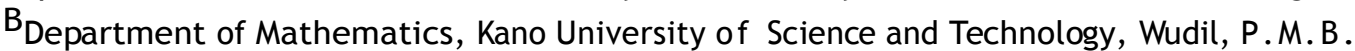 \\ 3244, Kano, Nigeria. \\ ${ }^{1}$ Corresponding author: nhussaini.mth@buk.edu.ng.
}

ABSTRACT

In this paper, we developed and qualitatively analyzed a model for the transmission dynamics of chikungunya virus in a human and mosquito populations. The chikungunya virus is transmitted by Aedes mosquitoes usually Aedes albopictus mosquito and it is of big threat to global public health. Rigorous analysis of the model shows that the model has a locally asymptotically stable disease free equilibrium whenever a certain epidemiological threshold quantity, called the basic reproduction number is less than unity (hence, the diseases would not persist in the community). The model has a unique endemic equilibrium when the reproduction number exceeds unity. Furthermore, the model exhibits the phenomenon of backward bifurcation, where the stable disease free equilibrium coexists with a stable endemic equilibrium.

Keywords: Chikungunya virus; vertical transmission; stability; backward bifurcation.

INTRODUCTION

Chikungunya virus (CHK) is viral disease that is transmitted to humans through the bites of an infected female mosquitoes (vector-bone disease) (Agusto et al., 2016), it is an arboviral disease caused by a member of the genus alphavirus that belongs to togaviridae family (Manore et al., 2014; Saswat et al., 2013; Naowarat et al., 2011; Yakob and Clements, 2013). CHK virus is a non-fatal disease that results in too much morbidity in an affected humans and has an effect on the country's economy as estimated by disability adjusted life years (DALY) thereby becoming a a big threat to global public health and development (Manore et al., 2014; Saswat et al., 2013; Jain et al., 2016). CHK virus gives permanent immunity after recovery of an individual (Manore et al., 2014; Saswat et al., 2013).

CHK virus is endemic in more than 60 countries in Asia, Africa, Europe, and America resulting several clinical cases or death (Saswat et al., 2013; Naowarat et al., 2011; Yakob and Clements, 2013; Dumont and Tchuenche, 2011; WHO, 2016). CHK was not considered as a life threatening disease because it has a low death rate (Manore et al., 2014). More than 1.3 million suspected cases of CHK and about 200 death cases have been reported in the Latin American countries, Caribbean islands, and the
United States of America in April, 2015 (Yakob and Clements, 2013; WHO, 2016).

The CHK virus is a vector bone diseases (transmitted to humans via the bites of an infected mosquitoes) (Manore et al., 2014). Aedes aegypti and Aedes albopictus mosquitoes are the principal vectors for the transmission of $\mathrm{CHK}$ virus worldwide and the viral infection has the following primary signs and symptoms in the patients which are fever, joint pain, headache, muscle pain, skin rash and incapacitating arthralgia (Manore et al., 2014; Saswat et al., 2013; Naowarat et al., 2011).

Vertical transmission has been shown to be an important component that enables the virus to be kept up in non-conducive climatic conditions in nature (Jain et al., 2016). Recently, experimental vertical transmission of $\mathrm{CHK}$ virus in Aedes aegypti has been shown (Agarwal et al., 2014). Information on vertical transmission of $\mathrm{CHK}$ virus in natural population is of great significance in order to comprehend the possible processes of virus survival during inter epidemic periods. In this paper we reported vertical transmission of $\mathrm{CHK}$ virus in aquatic and adult stages in the mosquito population.

Currently, there is no specific and effective vaccine for $\mathrm{CHK}$ virus at the moment, but a number of vaccines are undergoing clinical trials (Manore et al., 2014). 
Special Conference Edition November, 2017

Although there is no specific treatment for CHK virus (Manore et al., 2014; Saswat et al., 2013). However, treatment could be done by decreasing the symptoms in order to reduce the burden of the disease (WHO, 2016; Agusto et al., 2016).

A number of mathematical models have been designed to give insights into the transmission dynamics of CHK (see, for instance, (Manore et al., 2014; Saswat et al., 2013; Naowarat et al., 2011; Yakob and Clements, 2013; Dumont and Tchuenche, 2011; Agusto et al., 2016)). To the author's knowledge, the current study give the first model for CHK virus that incorporate the aquatic stage (egg, lava and pupa stages) and vertical transmission in a mosquito population.

\section{Model Formulation}

The total human population at time $t$, denoted by $N_{H}(t)$, is divided into four mutually exclusive compartments as follows: susceptible individuals, who are at risk of infection of $\mathrm{CHK}$ virus $\left(S_{H}(t)\right)$, asymptomatic CHK individuals $\left(E_{H}(t)\right)$, CHK early infected individuals with clinical symptoms of $\mathrm{CHK}\left(I_{H_{1}}(U)\right), \quad \mathrm{CHK}$ advance infected individuals with clinical symptoms of $\mathrm{CHK}\left(I_{H z}(t)\right)$, individuals who recovered from $\mathrm{CHK}\left(R_{H}(t)\right)$, so that:

$N_{H}(t)=S_{H}(t)+E_{H}(t)+I_{H 1}(t)+I_{H_{1}}(t)+R_{H}(t)$

Similarly, the total mosquito (female Aedes albopictos or Aedes aegypti mosquito) population at time $t$ denoted by $\left(N_{v}(t)\right)$, is sub-divided into sub-populations of immature mosquitoes (eggs, larvae and pupae stages), denoted by $A(t)$, and adult mosquitoes (denoted by $N_{I}(t)$, , so that: $N_{v}(t)=A(t)+N_{I}(t)$. where $\quad N_{I}(t)$ is further divided into three compartments as follows: adult mosquitoes susceptible to $\mathrm{CHK}$ viruses $\left(S_{v},(t)\right)$, adult mosquitoes exposed to CHK $\left(E_{w}(t)\right)$, CHK-infected adult mosquitoes $\left(I_{v}(\mathrm{t})\right.$, so that: $N_{l}(\mathrm{t})=S_{v}(\mathrm{t})+E_{v}(\mathrm{t})+I_{v}(\mathrm{t})$.

The susceptible population of individual who are at the risk of $\mathrm{CHK}$ virus infection $\left(S_{H}(t)\right)$ is generated by recruitment of humans at a constant rate $\Pi_{H}$ (all humans recruited into the population are assumed to be at risk of $\mathrm{CHK}$ infection). The population is diminished following infection with $\mathrm{CHK}$ (at a rate $\lambda_{H}$ ), and is also decreased by natural death (at a rate $\mu_{E}$ : this is assumed to be the same in all human compartments).

Thus, $\frac{d S_{H}}{d t}=\|_{H}-\lambda_{H} \mathbf{S}_{H}-\mu_{H} \mathbf{S}_{H}$.
The population of asymptomatic $\mathrm{CHK}$ individuals $\left(E_{H}\right)$ is generated following the infection of susceptible individuals (at a rate $\lambda_{1} H$ ) . It is decreased by the development of clinical symptoms of $\mathrm{CHK}$ (at a rate $\sigma_{1}$ ), and natural

Hence, $\frac{d E_{H}}{d t}=\lambda_{H} \mathbf{S}_{H}-\left(\sigma_{1}+\mu_{H}\right) \mathbf{E}_{H}$. death.

The population of $\mathrm{CHK}$ early infected humans with clinical symptoms of $\mathrm{CHK}\left(I_{H 1}\right)$ is generated by progression of CHK-exposed individuals (at the rate $\tau_{1}$ ). The population is decreased by progression to the advancedinfectious state of $\mathrm{CHK}$ (at a rate $\sigma_{\mathbf{z}}$ ). The population is further decreased by natural death and $\mathrm{CHK}$-induced death (at a rate $\hat{\delta}_{1}$ ). So that,

$\frac{d l_{H 1}}{d t}=\sigma_{1} \mathbf{E}_{H}-\left(\sigma_{2}+\delta_{1}+\mu_{H}\right) \mathbf{I}_{H 1}$.

The population of individuals at advancedinfectious state of CHK with clinical symptoms of $\mathrm{CHK}\left(\hat{l}_{\mathrm{Hz}}\right)$ is generated at the rate $\sigma_{\mathrm{z}}$. The population is decreased by recovery of CHK (at a rate $\left.\tau_{H}\right)$. It is further decreased by natural death and $\mathrm{CHK}$-induced death (at a rate $\delta_{2}$ ). So that,

$\frac{d l_{H 2}}{d t}=\sigma_{2} \mathbf{E}_{H}-\left(\tau_{H}+\delta_{2}+\mu_{H}\right) \mathbf{I}_{H 2}$.

The population of recovered $\mathrm{CHK}$-infected individuals $\left(\mathbb{R}_{H}\right)$ is generated by the successful treatment of CHK-infected individuals (at the rate $\tau_{H}$ ). It is diminished by natural death. Therefore, $\frac{d R_{H}}{d t}=\tau_{H} I_{H \mathbf{z}}-\mu_{H} \mathbf{R}_{H}$.

Immature mosquitoes (eggs, larvae and pupae) are lumped into a single compartment $(A)$ for computational convenience (Okuneye \& Gumel, 2016). The population of immature mosquitoes is generated at the rate $\left(\boldsymbol{\Pi}_{v}\right)$, where $\Pi_{v}$ is the egg deposition rate. The population of immature mosquitoes is decreased by maturation to susceptible adult mosquitoes (at a rate $\xi$ ), infectious adult mosquitoes (at a rate $(1-\xi)$ ) and natural death (at a rate $\mu_{A}$ ). So that,

$\frac{d A}{d t}=\Pi_{v}-\left(1+\mu_{A}\right) A$.

The population of susceptible adult mosquitoes $\left(S_{w}\right)$ is generated at the rate $(\xi)$. The population is diminished by infection, following effective contact with CHKinfected (at a rate $\lambda_{v}$ ) and natural death (at a rate $\mu_{w}$; this rate is assumed, for mathematical convenience, to be same for all the epidemiological classes for mosquitoes). 
Special Conference Edition November, 2017

Hence, $\frac{d S_{v}}{d t}=\xi A-\lambda_{v} \mathbf{S}_{v}-\boldsymbol{\mu}_{v} \mathbf{S}_{w}$.

The population of CHK-exposed adult mosquitoes $\left(E_{v}\right)$ is generated following infection with CHK (at a rate $\lambda_{v}$ ). It further decreased by the progression of $\mathrm{CHK}$ (at a rate $\sigma_{v}$ ), and natural death. Thus, $\frac{d E_{v}}{d t}=\lambda_{v} \mathrm{~s}_{v}-\left(\sigma_{v}+\mu_{v}\right) \mathrm{E}_{v}$.

The population of CHK-infected adult mosquitoes $\left(I_{v}\right)$ is generated at the rate $(1-\xi)$ and $\left(\sigma_{v}\right)$. It is further decreased by natural death. Thus, $\frac{d I_{v}}{d t}=(1-\xi) A+\left(\sigma_{v}+\mu_{v}\right) \mathbf{E}_{v}$. The model for the CHK is given by the following deterministic system of non-linear differential equations (a flow diagram of the model is depicted in Figure 1).

$$
\begin{aligned}
& \frac{d S_{H}}{d t}=\Pi_{H}-\lambda_{H} \mathbf{s}_{H}-\boldsymbol{\mu}_{H} \mathbf{s}_{H} \text {, } \\
& \frac{d E_{H}}{d t}=\lambda_{H} \mathbf{s}_{H}-\left(\sigma_{1}+\mu_{H}\right) \mathrm{E}_{H} \text {, } \\
& \frac{d I_{H 1}}{d t}=\sigma_{1} \mathbf{E}_{H}-\left(\sigma_{2}+\delta_{1}+\mu_{H}\right) \mathbf{I}_{H 1} \text {. } \\
& \frac{d I_{H 2}}{d t}=\sigma_{2} \mathbf{E}_{H}-\left(\tau_{H}+\delta_{2}+\mu_{H}\right) \mathrm{I}_{H 2} \text {. } \\
& \frac{d R_{H}}{d t}=\tau_{H} I_{H z}-\mu_{H} \mathbf{R}_{H \prime}(1) \\
& \frac{d A}{d t}=\Pi_{v}-\left(1+\mu_{A}\right) A \text {, } \\
& \frac{d S_{v}}{d t}=\xi A-\lambda_{v} \mathbf{S}_{v}-\mu_{v} \mathbf{S}_{v} \text {. } \\
& \frac{d E_{v}}{d t}=\lambda_{v} \mathbf{S}_{v}-\left(\sigma_{v}+\mu_{v}\right) \mathbf{E}_{v} \text {, } \\
& \frac{d I_{v}}{d t}=(1-\xi) A+\sigma_{v} \mathbf{E}_{v}-\boldsymbol{\mu}_{v} I_{v} \\
& \text { where, } \lambda_{H}=\frac{\beta_{H} \sigma_{m} \sigma_{H}}{\left(\sigma_{m 2} N_{v}+\sigma_{H} N_{H}\right)\left(\eta_{v} E_{v}+I_{v}\right)} \text { and } \quad \lambda_{v}=\frac{\beta_{H} \sigma_{m} \sigma_{H}}{\left(\sigma_{m} N_{v}+\sigma_{H} N_{H}\right)\left(\eta_{H} E_{H}+\eta_{11} I_{H 1}+\eta_{2} I_{H 2}\right)}
\end{aligned}
$$

, (2)

$N_{H}(t)=S_{H}(t)+E_{H}(t)+I_{H 3}(t)+I_{H 2}(t)+F_{H}(t)$ and $N_{v}(t)=A+S_{v}(t)+E_{v}(t)+I_{v}(t)$

In (2), $\beta_{H}$ is the transmission probability of $\mathrm{CHK}$ and $\eta_{H}, \eta_{1}, \eta_{n}, \eta_{v}>1$ are the modification parameters accounting for the assumption that infected humans and mosquitoes are more infectious than exposed humans and mosquitoes, respectively (Duong et al., 2015). Furthermore, $h_{1}\left(N_{l l}, N_{v}\right)$ is the per capita biting rate of female mosquito on the human host per unit time. Similarly, $b_{2}\left(N_{H}, N_{w}\right)$ is the number of bites per mosquito per unit time. Following (Chitnis et al., 2006), the biting rates $b_{1}\left(N_{H}, N_{v}\right)$ and $b_{2}\left(N_{H}, N_{v}\right)$ are respectively given by,

$$
b_{1}\left(N_{H}, N_{v}\right)=\frac{\sigma_{m} \sigma_{H} N_{v}}{\sigma_{m} N_{w}+\sigma_{H} N_{H}} \text { and } b_{z}\left(N_{H}, N_{v}\right)=\frac{\sigma_{m} \sigma_{H} N_{H}}{\sigma_{m} N_{w}+\sigma_{H} N_{H}}
$$

Where $\sigma_{m}$ is the number of times one mosquito would want to bite humans per unit time (if humans were freely available) and $\sigma_{H}$ is the maximum number of mosquito bites a human can receive per unit time.
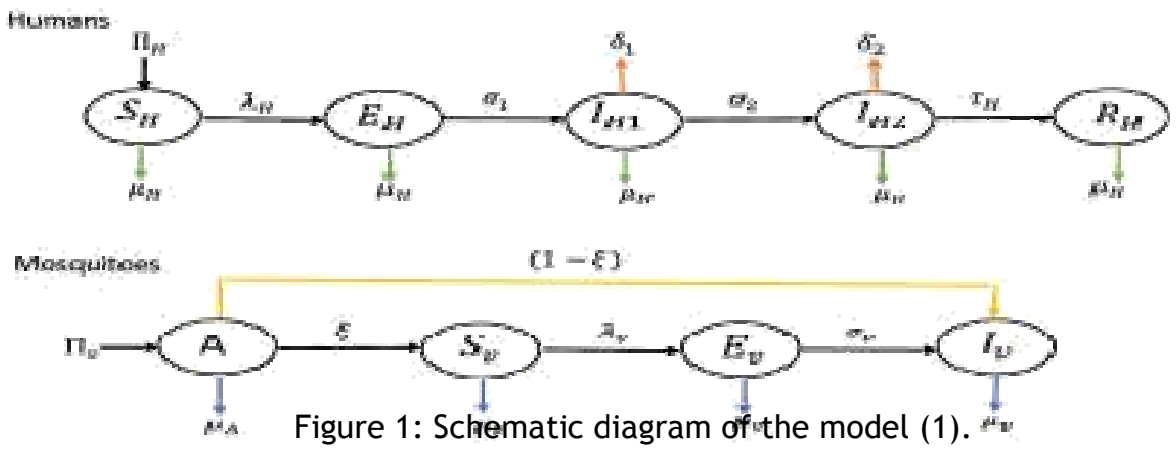


\section{Special Conference Edition November, 2017}

The model (1) is an extension of some of the CHK transmission models (e.g., those in (Manore et al., 2014; Yakob \& Clements, 2013; Agusto et al., 2016) by (inter alia) :

i. Including the dynamics of early infectious and advanced infectious state of $\mathrm{CHK}$ in human population (this was not included in (Agusto et al., 2016; Yakob \& Clements, 2013));

ii. Using a nonlinear biting rate (constant rate was used in (Agusto et al., 2016);

iii. Including the dynamics of an aquatic (immature) stage of the mosquito (egg, lava and pupa stages) (this was not considered in (Manore et al., 2014; Yakob \& Clements, 2013; Agusto et al., 2016)).

Basic qualitative properties of the model

In this section, the basic qualitative features of the model (1) will now be explored.

Let $\mu=\min \left(\mu_{A}, \mu_{v}\right)$. We claim the following:

Theorem: The system (1) preserves positivity of solutions. In other words, the solutions of the

model (1) with positive initial data and remain positive $\forall t>0$. Further, $\lim _{t \rightarrow+\infty} S u p N_{H} \leq \frac{\Pi_{H}}{\mu_{H}}$ and $\lim _{t \rightarrow \mathbf{m}} \operatorname{Sup}_{v} \leq \frac{\mathbf{\Pi}_{v}}{\mu}$.

Proof. It is clear from the first equation of the model (1) that,

$$
\frac{d S_{H}}{d t}=\eta_{H}-\lambda_{H} \mathbf{s}_{H}-\mu_{H} \mathbf{s}_{H} \geq-\left(\lambda_{H}+\mu_{H}\right) \mathbf{s}_{H}
$$

so that, $S_{H}(t) \geq \mathbf{S}_{H}(\mathbf{0}) \exp \int^{-\int_{0}^{t}\left(\lambda_{H}+\mu_{H}\right) d t}>0 .(4)$

Using similar approach, it can be shown that all other state variables of the model remain positive $\forall t>0$. Furthermore, adding the first five equations in the model (1) and the last four equations of the system (1) gives, respectively,

$$
\frac{d N_{H}(t)}{d t}=\Pi_{H}-\mu_{H} N_{H}-\delta_{1} I_{H 1}-\delta_{\mathbf{z}} I_{H z}(5)
$$

and $\frac{d N_{v}(t)}{d t}=\Pi_{v}-\mu_{A} A-\mu_{v} N_{I}(6)$

Thus,

$\Pi_{v}-\mu_{A} A-\mu_{v} N_{I} \leq \frac{d N_{v}(t)}{d t} \leq \Pi_{v}-\mu N_{v}$

$\Pi_{H}-\mu_{H} N_{H}-\hat{o}_{1} I_{H 1}-\delta_{2} I_{H 2} \leq \frac{d N_{H}(t)}{d t} \leq \Pi_{H}-\mu_{H} N_{H}$ and

$\frac{\Pi_{H}}{\mu_{H}+\delta_{1}+\delta_{2}} \leq \lim _{t \rightarrow \infty} \inf N_{H}(t) \leq \lim _{t \rightarrow \infty} \sup N_{H}(t) \leq \frac{\Pi_{H}}{\mu_{H}}$ and

$\frac{\Pi_{v}}{\mu_{A}+\mu_{v}} \leq \lim _{t \rightarrow \infty} \inf N_{v}(t) \leq \lim _{t \rightarrow \infty} \sup N_{v}(t) \leq \frac{\Pi_{v}}{\mu} . \quad$ Hence, $\quad \lim _{t \rightarrow x} \sup N_{H}(t) \leq \frac{\Pi_{H}}{\mu_{H}}$ and

$\lim _{t \rightarrow \infty} \sup N_{v}(t) \leq \frac{\Pi_{v}}{\mu}$.

Lemma:The following biologically feasible region of the model equation (1)

$\mathrm{D}=\left\{\left(S_{H}, E_{H}, I_{H 1}, I_{H 2}, R_{H}, A, S_{v}, E_{v}, I_{v}\right) \in \mathbf{R}_{+}^{9}: N_{H} \leq \frac{\Pi_{H}}{\mu_{H}} N_{v} \leq \frac{\Pi_{v}}{\mu}\right\}_{i s}$ positively invariant and attracting. Refer to (Van-den \& Watmough,2002) for the proof of the above lemma. Therefore, the model (1) is mathematically well-posed and epidemiologically reasonable since all the variables remain non-negative for all $t \geq 0$. Hence, it is sufficient to consider the dynamics of the model (1)in $D$ (Hethcote,2000).

Asymptotic stability of disease-free equilibrium (DFE)

\section{TDFE}

Theorem: The TDFE of the model (1), denoted by $Y_{0}$, is GAS in $\Omega$ whenever $R_{N} \leq 1$.

Proof. Following(Okuneye\&Gumel,2016), the model(1)can be re-written as $\frac{d Y}{d t}=A(Y) Y+G$, 
Where $Y=\left(S_{H}, E_{H}, I_{H 1}, I_{H z}, R_{H}, A, S_{v,} E_{w}, I_{v}\right)^{T}, A(Y)$ isa9x9M-matrix(Metzler-Matrix)given by,

\begin{tabular}{|c|c|c|c|c|c|c|c|c|c|}
\hline \multirow{3}{*}{\multicolumn{2}{|c|}{$\left[\begin{array}{c}\frac{-\beta_{H} \sigma_{m} \sigma_{H}\left(\eta_{v} E_{v}+I_{v}\right)}{\sigma_{m} N_{v}+\sigma_{H} N_{H}}-\mu_{H} \\
\frac{\beta_{H} \sigma_{m} \sigma_{H}\left(\eta_{v} E_{v}+I_{v}\right)}{\sigma_{m} N_{v}+\sigma_{H} N_{H}} \\
0\end{array}\right.$}} & $-\tau-\mu$ & 0 & 0 & 0 & 0 & 0 & $\frac{-\beta_{H} \sigma_{m} \sigma_{H} \eta_{v} S_{H}}{\sigma_{m} N_{v}+\sigma_{H} N_{H}}$ & $\frac{-\beta_{H} \sigma_{m} \sigma_{H} S_{H}}{\sigma_{m} N_{v}+\sigma_{H} N_{H}}$ \\
\hline & & $-\sigma_{H}-\mu_{H}$ & 0 & 0 & 0 & 0 & 0 & $\frac{\sigma_{m} N_{v}+\sigma_{H} N_{H}}{\sigma_{H}}$ & $\sigma_{m} N_{v}+\sigma_{H} N_{H}$ \\
\hline & & $\sigma_{1}$ & 0 & 0 & 0 & 0 & 0 & 0 & \\
\hline \multirow{5}{*}{$A(Y)=$} & 0 & 0 & 0 & 0 & 0 & 0 & 0 & 0 & 0 \\
\hline & 0 & 0 & 0 & 0 & $-\mu_{H}$ & 0 & 0 & 0 & 0 \\
\hline & 0 & 0 & 0 & 0 & 0 & $-1-\mu_{A}$ & 0 & 0 & 0 \\
\hline & 0 & $\frac{-\beta_{v} \sigma_{m} \sigma_{H} \eta_{H} S_{v}}{\sigma_{m} N_{v}+\sigma_{H} N_{H}}$ & $\frac{-\beta_{v} \sigma_{m} \sigma_{1} \eta_{H} S_{v}}{\sigma_{m} N_{v}+\sigma_{H} N_{H}}$ & $\frac{-\beta_{v} \sigma_{m} \sigma_{1} \eta_{2} S_{v}}{\sigma_{m} N_{v}+\sigma_{H} N_{H}}$ & 0 & $\xi$ & $\frac{-\beta_{v} \sigma_{m} \sigma_{H}\left(\eta_{H} E_{H}+\eta_{1} I_{H 1}+\eta_{2} I_{H 2}\right)}{\sigma_{m} N_{v}+\sigma_{H} N_{H}}-\mu_{H}$ & 0 & 0 \\
\hline & $\begin{array}{l}0 \\
0\end{array}$ & $\frac{\beta_{v} \sigma_{m} \sigma_{H} \eta_{H} S_{v}}{\sigma_{m} N_{v}+\sigma_{H} N_{H}}$ & $\frac{\beta_{v} \sigma_{m} \sigma_{1} \eta_{H} S_{v}}{\sigma_{m} N_{v}+\sigma_{H} N_{H}}$ & $\frac{\beta_{v} \sigma_{m} \sigma_{1} \eta_{2} S_{v}}{\sigma_{m} N_{v}+\sigma_{H} N_{H}}$ & 0 & $\begin{array}{c}0 \\
1-\xi\end{array}$ & $\frac{\beta_{v} \sigma_{m} \sigma_{H}\left(\eta_{H} E_{H}+\eta_{1} I_{H 1}+\eta_{2} I_{H 2}\right)}{\sigma_{m} N_{v}+\sigma_{H} N_{H}}$ & $-\sigma_{v}-\mu_{v}$ & 0 \\
\hline
\end{tabular}

And $G=\left(\boldsymbol{\Pi}_{H}, 0,0,0,0,0,0,0,0\right)^{T}$. let $R_{N}<1$ (so the model (1) has only the TDFE, $\tau_{0}$ ). Furthermore, let $Z=Y-T D F E$. Thus, the equation $\frac{d Y}{d t}=A(Y) Y+G$ can be re-written as $\frac{d Z}{d t}=B(Z) Z$, where $B(Z)$ is the $9 \times 9$ matrix of coefficients of the model (1) with variables $Z_{i}(i=1,2,3,4,5,6,7,8,9)$. It is clear that $T D F E_{Z}=(0,0,0,0,0,0,0,0,0)$ is the only equilibrium of the system $\frac{d Z}{d t}=B(Z) Z$. Consider the Lyapunov function $V(Z)=\langle W, Z\rangle$ with $W=\left(\frac{1,1,1,1,1,1}{\Pi_{v}}, \frac{1}{\mu_{v}}, \frac{1}{\mu_{v}}, \frac{1}{\mu_{v}}\right) \gg 0$

(Okuneye \& Gumel, 2016). Thus $V(Z)>0$ except at $Z=T D F E_{Z}$. Furthermore (where a prime now denotes differentiation with respect to $Z$ ), $V^{z}(Z)=\langle W, B(Z) Z\rangle=-\left(Z_{7}+Z_{9}+Z_{9}\right)-\frac{1+\mu_{A}}{\Pi_{v}} Z_{6}+\frac{1}{\mu_{v}} Z_{6}=-\left(Z_{7}+Z_{8}+Z_{9}\right)-\frac{1+\mu_{A}}{\Pi_{v}\left(1-R_{N}\right) Z_{6}}$

Since $R_{N} \leq 1$ in $C\left([0], \mathbf{R}_{+}^{9}\right)$, it follows that $V^{x}(Z) \leq 0$. Furthermore, it follows from La Salles's Invariance Principle (Theorem 6.4 of (LaSalle, 1976)) that the maximal invariant set contained in $\frac{V}{V^{\prime}(Z)} \leq \mathbf{0}$ is the TDFE $E_{Z}$. Thus, the transformed equilibrium, TDFE, is GAS in C([0], $\left.\mathbf{R}_{m}^{9}\right)$ if $R_{N} \leq 1$.

NDFE

Using the next generation operator method on the system(Van-den\&Watmough,2002), the associated basic reproduction number of the model (9), denoted by $R_{0}$, is given by,

$$
\begin{aligned}
& R_{0}=\sqrt{\frac{\left.g \Pi_{v} \Pi_{H} \beta_{v} \beta_{c} \mu_{H}\left(\eta_{v} \mu_{v}+\sigma_{v}\right)\left(\eta_{H} \mathbf{Q}_{2}+\eta_{1} \sigma_{1}\right) \mathbf{Q}_{3}+\eta_{2} \sigma_{1} \sigma_{2}\right) \sigma_{H}^{2} \sigma_{m}^{2}}{\mathbf{Q}_{1} \mathbf{Q}_{2} \mathbf{Q}_{3} \mathbf{Q}_{4}\left(\mathbf{1}+\mu_{A}\right) \mu_{v}^{2}\left(\mu_{H} \sigma_{m} \Pi_{v}+\mu \sigma_{H} \Pi_{H}\right)^{2}}}, \text { where, } \\
& C_{1}=\frac{\Pi_{H} \beta_{c} \sigma_{m} \sigma_{H} \mu}{\mu_{H} \sigma_{m} \Pi_{v}+\mu \sigma_{H} \Pi_{H}}, \quad C_{2}=\frac{\Pi_{v} \xi \beta_{v} \sigma_{m} \sigma_{H} \mu \mu_{H}}{\mu_{2}\left(1+\mu_{A}\right)\left(\mu_{H} \sigma_{m} \eta_{v}+\mu \sigma_{H} \Pi_{H}\right)}, \quad Q_{1}=\sigma_{1}+\mu_{H}, \\
& Q_{2}=\sigma_{2}+\delta_{1}+\mu_{H}, Q_{3}=\tau_{H}+\delta_{2}+\mu_{H}, \text { and } Q_{4}=\sigma_{v}+\mu_{v} .
\end{aligned}
$$

Lemma: The DFE $\left(\bar{E}_{0}\right)$, of the CHK model(1) is locally asymptotically stable (LAS) if $R_{0} \approx 1$, and unstable if $R_{0}>1$.

Existence of backward bifurcation

Here, the center manifold theory will be use to investigate the conditions on the parameter values in the model that cause forward or backward bifurcation to occur (Carr,1981;CastilloChavez\&Song,2004; Van-den \& Watmough,2002).

Consider the system, $\frac{d x}{d t}=f(x, \psi)$, where $\psi$ is the bifurcation parameter, $f$ is continuously differentiable at least twice in both $x$ and $\psi$. The disease-free equilibrium is the line $\left(x_{0}, \psi\right)$ and the local stability of the disease-free equilibrium at the point $\left(x_{0}, \psi\right)$ (Van-den \& Watmough, 2002). Now it shall show that there are non-trivial equilibrium near the bifurcation parameter.

$$
\begin{aligned}
& \text { Solving for } R_{\mathrm{a}}=\mathbf{1} \text { gives } \\
& \beta_{C}=\beta_{C}^{*}=\frac{\mathbf{Q}_{1} \mathbf{Q}_{2} \mathbf{Q}_{3} \mathbf{Q}_{4}\left(\mathbf{1}+\mu_{A}\right) \mu_{v}^{2}\left(\mu_{H} \sigma_{m} \Pi_{v}+\mu \bar{\sigma}_{H} \Pi_{H}\right)^{2}}{\left.\xi \Pi_{v} \Pi_{H} \beta_{v} \beta_{c} \mu_{H}\left(\eta_{v} \mu_{v}+\sigma_{v}\right)\left(\eta_{h} \mathbf{Q}_{2}+\eta_{1} \sigma_{1}\right) \mathbf{Q}_{3}+\eta_{2} \sigma_{L} \sigma_{2}\right) \sigma_{H}^{2} \sigma_{m}^{2}} .
\end{aligned}
$$




\section{Special Conference Edition November, 2017}

By Lemma 3.3, the disease-free equilibrium $\boldsymbol{\varepsilon}_{0}$ is locally stable when $\beta_{\varepsilon}<\beta_{\varepsilon}^{*}$ and unstable when $\beta_{c}=\beta_{c}^{*}$. Here $\beta_{c}-\beta_{c}^{*}$ is the bifurcation value. For convenience, let $S_{H}=x_{1}, E_{H}=x_{2}$, $I_{H 1}=x_{3}, \quad I_{H 2}=x_{4}, \quad R_{H}=x_{5}, \quad A=x_{6}, \quad S_{v}=x_{7}, \quad E_{v}=x_{3}, \quad I_{v}=x_{9}$, so that $N_{H}(t)=x_{1}+x_{2}+x_{3}+x_{4}+x_{5}$ and $N_{v}(t)=x_{6}+x_{7}+x_{\mathrm{g}}+x_{9}$.Further, by adopting the same vector notations with $x=\left(x_{2}, x_{2}, \ldots x_{0}\right)^{T}$, the model (1) can be written in the form $\frac{d x}{d t}=F(x)$ where $f=\left(f_{1}, f_{2}, \ldots f_{9}\right)^{T}$.

The Jacobian of the transformed system, evaluated at the DFE $\left(E_{0}\right)$ with $\beta_{C}-\beta \bar{c}$ (denoted by $\left(E_{\circ}\right)$ ), is given by

$$
J\left(E_{0}\right)=\left[\begin{array}{ccccccccc}
-\mu_{H} & 0 & 0 & 0 & 0 & 0 & 0 & -s_{1} & -s_{2} \\
0 & -Q_{1} & 0 & 0 & 0 & 0 & 0 & s_{1} & s_{1} \\
0 & \sigma_{1} & -Q_{2} & 0 & 0 & 0 & 0 & 0 & 0 \\
0 & 0 & \sigma_{2} & -Q_{3} & 0 & 0 & 0 & 0 & 0 \\
0 & 0 & 0 & \tau_{H} & -\mu_{H} & 0 & 0 & 0 & 0 \\
0 & 0 & 0 & 0 & 0 & -Q_{5} & 0 & 0 & 0 \\
0 & -s_{3} & -s_{4} & -s_{5} & 0 & \xi & -\mu_{v} & 0 & 0 \\
0 & s_{3} & s_{4} & s_{5} & 0 & 0 & 0 & -Q_{4} & 0 \\
0 & 0 & 0 & 0 & 0 & Q_{6} & 0 & \sigma_{v} & -\mu_{v}
\end{array}\right]
$$

where

$$
S_{1}=\frac{\beta_{H} \sigma_{m} \sigma_{H} \eta_{v} \Pi_{H} \mu_{v}\left(1+\mu_{A}\right)}{\left(\Pi_{H}\left(1+u_{A}\right) \sigma_{H}+\sigma_{m} \Pi_{v} \mu_{H}\right) \mu_{v}+\sigma_{m} \Pi_{w} \mu_{H} \xi}
$$

$S_{2}=\frac{\beta_{H} \sigma_{m} \sigma_{H} \Pi_{H} \mu_{v}\left(1+\mu_{A}\right)}{\left(\Pi_{H}\left(1+\mu_{A}\right) \sigma_{H}+\sigma_{m} \Pi_{v} \mu_{H}\right) \mu_{v}+\sigma_{m} \Pi_{v} \mu_{H} \xi}$,

$S_{3}=\frac{\beta_{v} \sigma_{m} \sigma_{H} \eta_{H} \xi \Pi_{v} \mu_{H}}{\sigma_{m} \Pi_{v}\left(\mu_{v}+\xi\right)_{\mu_{H}}+\sigma_{H} \Pi_{H} \mu_{v}\left(1+\mu_{A}\right)}, S_{4}=\frac{\rho_{v} \sigma_{m} \sigma_{H} \eta_{1} \eta_{i} \Pi_{v} \mu_{H}}{\sigma_{m} \Pi_{v}\left(\mu_{v}+\xi\right)_{\mu_{H}}+\sigma_{H} \Pi_{H} \mu_{v}\left(1+\mu_{A}\right)}$

$S_{5}=\frac{\beta_{v} \sigma_{m} \sigma_{H} \eta_{2} \xi \Pi_{v} \mu_{H}}{\sigma_{m} \Pi_{v}\left(\mu_{v}+\xi\right) \mu_{H}+\sigma_{H} \Pi_{H} \mu_{v}\left(1+\mu_{A}\right)}$

The Jacobian $J\left(E_{\mathrm{n}}\right)$ of the linearized system has a simple zero eigen value (with all other eigen values having negative real part). Using the notation in (Castito-Chavez \& Song, 2004), the following computations are carried out.

Eigen vectors of $/\left(E_{0}\right)_{\beta_{C}=\beta_{C}}$ : For the case when $R_{0}=\mathbf{1}$ it can be shown that the $J\left(E_{0}\right)$ has a right eigen vector (Corresponding to the zero eigen value), given by $w=\left[w_{1}, w_{2}, \ldots, w_{9}\right]^{T}$ where, $w_{1}=\frac{-1}{\mu_{H}}\left[\frac{S_{1} \mu_{v}\left(S_{3} Q_{2} Q_{3}+\sigma_{1} S_{4} Q_{3}+\sigma_{1} \sigma_{2} S_{5}\right)+S_{2} \sigma_{v}\left(S_{3} Q_{2} Q_{3}+\sigma_{1} S_{4} Q_{3}+\sigma_{1} \sigma_{3} S_{5}\right)}{\sigma_{1} \sigma_{2} Q_{4} \mu_{v}}\right] w_{4}$,

$$
\begin{aligned}
& w_{2}=\frac{Q_{2} Q_{3}}{\sigma_{2} \sigma_{2}} w_{4}, \quad w_{4}=\frac{Q_{3}}{\sigma_{2}} w_{4}, \quad w_{4}>0, \quad w_{5}=\frac{\tau_{H}}{\mu_{H}} w_{4}, \quad w_{6}=0 \text {, } \\
& w_{7}=-\frac{S_{3} Q_{2} Q_{3}+\sigma_{1} S_{4} Q_{3}+\sigma_{1} \sigma_{2} S_{5}}{\sigma_{2} \sigma_{2} \mu_{y}} w_{4}, \quad w_{3}=\frac{S_{3} Q_{2} Q_{3}+\sigma_{1} S_{4} Q_{3}+\sigma_{1} \sigma_{2} S_{5}}{\sigma_{1} \sigma_{2} Q_{4}} w_{4},
\end{aligned}
$$

$w_{9}=\frac{S_{3} Q_{2} Q_{3}+\sigma_{1} S_{4} Q_{3}+\sigma_{1} \sigma_{2} S_{5}}{\sigma_{1} \sigma_{2} \mu_{2} Q_{4}} \sigma_{v} w_{4}$. Similarly, the components of the left eigenvector of $J\left(E_{\mathrm{n}}\right)$ (corresponding to the zero eigen value), denoted by $v=\left[v_{1}, v_{2}, \ldots, v_{q}\right]$, are given by, 
Special Conference Edition November, 2017

$v_{1}=0, v_{2}=\frac{\sigma_{2} \sigma_{2} S_{5}+\sigma_{2} S_{4} Q_{3}+S_{3} Q_{2} Q_{3}}{Q_{1} Q_{2} S_{5}} v_{4}$
$v_{6}=\frac{\sigma_{1} \sigma_{2} S_{2} S_{5}+\sigma_{2} S_{2} S_{4} Q_{3}+S_{2} Q_{2} Q_{3}}{Q_{1} Q_{2} S_{5}} Q_{6} v_{4}$,
$v_{9}=\frac{\sigma_{1} \sigma_{2} S_{2} S_{5}+\sigma_{1} S_{2} S_{4} Q_{3}+S_{2} Q_{2} Q_{3}}{Q_{2} Q_{2} S_{5}} v_{4}$.

It is worth mentioning that the free right eigenvectors, $v_{*}$ and left eigenvector, $v_{\boldsymbol{*}}$, are chosen to be $v_{4}=1$

and

$w_{4}=\frac{1}{A_{1}+A_{2}}$, where,

$A_{1}=\frac{\left(\sigma_{1} \sigma_{2} S_{5}+\sigma_{1} S_{4} Q_{3}+S_{3} Q_{2} Q_{3}\right) Q_{2} Q_{3}+\left(\sigma_{2} S_{5}+S_{4} Q_{3}\right) Q_{1} \sigma_{1} Q_{3}+Q_{1} Q_{2} \sigma_{1} \sigma_{2} S_{5}}{Q_{1} Q_{2} S_{3} \sigma_{1} \sigma_{2}}$

$A_{2}=\frac{Q_{1} Q_{2} S_{4} \mu_{2}\left(S_{3} Q_{2} Q_{3}+\sigma_{1} S_{4} Q_{3}+\sigma_{1} \sigma_{2} S_{5}\right)+\left(\sigma_{1} \sigma_{2} S_{2} S_{5}+\sigma_{1} S_{2} S_{4} Q_{3}+S_{2} Q_{2} Q_{3}\right)\left(S_{3} Q_{2} Q_{3}+\sigma_{1} S_{4} Q_{3}+\sigma_{1} \sigma_{2} S_{5}\right) \sigma_{V} S_{5}}{Q_{1} Q_{2} Q_{3} S_{4} S_{5} \sigma_{1} \sigma_{2} \mu_{2}}$

, so that $v . w=1$ (in line with (Castillo-Chavez \& Song,2004)).It can be shown, by computing the non-zero partial derivatives of the right-hand side functions, $f_{i}(i=1, \ldots, 9)$, that the associated backward bifurcation coefficients, $\mathrm{a}$ and $\mathrm{b}$, are given, respectively, by(seeTheorem4.1in(Castillo-Chavez \& Song,2004)):

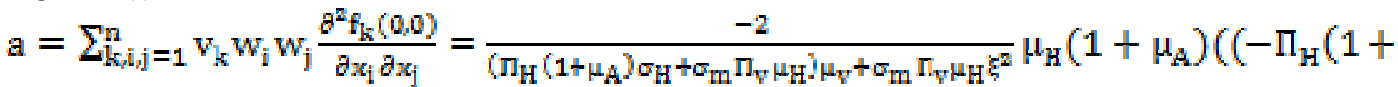

$$
\begin{aligned}
& \left.\mu_{\mathrm{A}}\right)\left(\mathrm{v}_{2}\left(w_{9}+w_{8} \eta_{\mathrm{v}}\right)\left(w_{2}+w_{3}+w_{4}+w_{5}\right) \beta_{\mathrm{H}}-v_{3} w_{7} \beta_{\mathrm{v}}\left(\eta_{1} w_{3}+\eta_{\mathrm{H}} w_{2}+\eta_{2} w_{4}\right)\right) \sigma_{H} \\
& +\left(-v_{2} \beta_{H}\left(1+\mu_{A}\right)\left(w_{y}+w_{y} \eta_{V}\right)\right. \\
& \left.\left.\left(w_{7}+w_{s}+w_{y}\right) \Pi_{H}+\Pi_{V} \mu_{H}\left(v_{L} w_{1}\left(w_{y}+w_{s} \eta_{V}\right) \beta_{k}+v_{s} w_{7} \beta_{v}\left(\eta_{2} w_{s}+w_{L} \eta_{H}+\eta_{L} w_{4}\right)\right)\right) \sigma_{m}\right)
\end{aligned}
$$

$\mu_{\mathrm{V}}+$

$\Pi_{V} \mu_{\mathrm{H}}\left(-v_{\mathrm{g}} \beta_{\mathrm{v}}\left(\eta_{\mathrm{I}} w_{\mathrm{s}}+w_{2} \eta_{\mathrm{H}}+\eta_{2} w_{4}\right)\left(w_{1}+w_{2}+w_{\mathrm{s}}+w_{4}+w_{\mathrm{b}}\right) \sigma_{H}+\sigma_{\mathrm{m}}\left(\mathrm{v}_{2} w_{1}\left(w_{4}+\right.\right.\right.$

$\left.\left.\left.\left.\mathrm{w}_{\mathrm{g}} \eta_{\mathrm{V}}\right) \beta_{\mathrm{H}}-\mathrm{v}_{\mathrm{g}} \beta_{\mathrm{v}}\left(\mathrm{w}_{\mathrm{g}}+\mathrm{w}_{9}\right)\left(\eta_{1} \mathrm{w}_{3}+\eta_{\mathrm{H}} \mathrm{w}_{2}+\eta_{\mathrm{Z}} \mathrm{w}_{4}\right)\right)\right) \xi\right) \mu_{\mathrm{V}} \sigma_{\mathrm{m}} \sigma_{\mathrm{H}}$

and $b=\sum_{k, i=1}^{n} v_{k} w_{i} \frac{\partial^{2} f_{k}(0,0)}{\partial x_{i} \partial \beta_{v}^{*}}=\frac{\left(\eta_{1} w_{3}+\eta_{H} w_{2}+\eta_{2} w_{4}\right) \Pi_{v} \xi_{\mu_{H}} \sigma_{H} \sigma_{m} v_{g}}{\left(\sigma_{m} \Pi_{v}\left(\mu_{v}+\xi\right) \mu_{H}+\sigma_{H} \Pi_{H} \mu_{v}\left(1+\mu_{A}\right)\right)}$

$R_{0}=1$

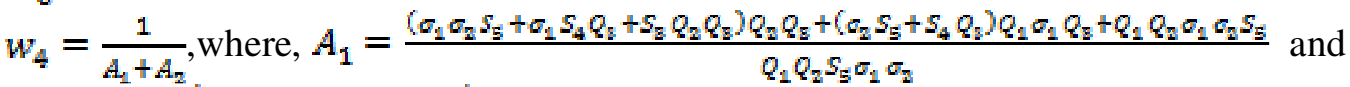

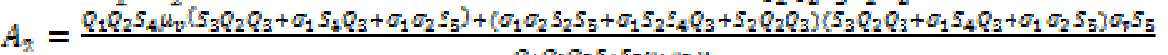

Since the coefficient $b$ is automatically positive, it follows that the model (1) will undergo backward bifurcation if the coefficient $a$, is positive.

\section{CONCLUSION}

This paper presents a deterministic model for the transmission dynamics of chikungunya. The model, which realistically adopts a standard incidence formulation, allows chikungunya transmission by exposed humans and mosquitoes. The model is rigorously analyzed to gain insights into the qualitative dynamics of chikungunya. The main theoretical and epidemiological findings of the study show that:

\section{REFERENCES}

Agarwal, A., Dash, P.K., Singh, A.K., Sharma,S., Gopalan,N., Rao,P.V.L., Parida,M.M., Re it e r, P.(2014). Evidence of experimental vertical transmission of emerging Novel ECSA genotype of Chikungunya virus in Aedes aegypti. PLoS Negl.Trop.Dis., 2990(8).
- The TDFE of the model is GAS whenever the threshold quantity is less than or equal to one.

- The model exhibits the phenomenon of backward bifurcation, where the stable NDFE co-exists with a stable endemic equilibrium, even when the reproduction number is less than unity.

Agusto, F.B., Easley,S., Freeman,K., Thomas, M. (2016). Mathematical model of three Age-Structured transmission dynamics of Chikungunya virus. J. Computational and Mathematical Methods in Medicine. http://doi.org/10.1155/2016/4320514. 
Special Conference Edition November, 2017

Carr,J.(1981). Application Centre Manifold Theory. Springer-verlag: New York.

Castillo-Chavez, C. Song, B. (2004). Dynamical model of tuberculosis and their applications. Math. Biosci.Eng., 1(2),361404.

Chitnis, N., Cushing, J.M., $\quad$ Hyman,J .M.(2006).Bifurcation analysis of a mathematical model for malaria transmission,SIAMJ.Appl.Math.,67(1),2445.

Dumont, Y., Tchuenche, J.M.(2011). Mathematical studies on the sterile insect technique for the Chikungunya disease and aedes albopictus. J. Math. Biol. http://dio.10.1007/s00285-0110477-6.

Duong,V., Lambrechts,L., Paul,R.E., Ly,S., Lay, R.S., Long,K.C., Huy, R., Tarantola, A., Scott, T.W., Sakuntabhai, A., Buchy, P. (2015). Asymptomatic humans transmit dengue virus to mosquitoes. Proc Natl Acad Sci USA, 112(47),14688-14693.http://doi:1 $0.1073 /$ pnas. 1508114112 .

Garba,S .M., Gumel, A.B., Bukar, M.R.A.(2008). Backward bifurcations in Dengue transmission dynamics. Math.Biosci. 215,11-25.

Hethcote, H.W. (2000). The Mathematics of infectious diseases. SIAMRev., 42,599-653.

Hussaini, N., Lubuma, J. M-S., Barley,K., Gumel, A.B.(2016). Mathematical analysis of a model for AVL-HIV coendemicity. Mathematical Biosciences 271,80-95.

Jain, J., Kushwaha, R.B.S., Singha,S.S., Sharmaa, A., Adakb,T., Singh,Om P., Kamal,R.B., Subbaraoc, S.K., Sunil, S.(2016). Evidence for natural vertical transmission of Chikungunya viruses in field populations of Aedes aegypti in Delhi and Haryana states in India preliminary report. ActaTropica, 162,46-55.

LaSalle,J.P.(1976). The stability of dynamical systems. Regional Conference Series in Applied Mathematics. SIAM Philadephia.

Manore, Hickman, K.S., Xu, S., Wearing,H.J., Hyman, J.M.(2014).Comparing Dengue and Chikungunya emergence and endemic transmission in A. aegypti and A. albopictus. J.Theor.Bio., 356,174-191.

Naowarat, S., Tawarat,W., Tang,I.M. (2011). Control of the transmission of Chikungunya fever epidemic through the use of adulticide. American Journal of Applied Sciences, 8(6),558-565.
Okuneye, K., Gumel, A.B.(2016). Analys is of a temperature-and rainfall- dependent model for malaria transmission dynamics. Mathematical Biosciences 000,121.http://dx.doi.org/10.1016/j.mbs. 20 16.03.013.

Saswat,T., Kumar, A., Kumar,S., Mamidi,P., Muduli,S., Debata,N. K., Pal, N.S., Pratheek, B.M., Chattopadhyay, S.Chattopadhyay, S. (2015). High rates of co-infection of Dengue and Chikungunya virus in Odisha and Maharashtra, India during 2013. Infectious, GeneticsandEvolution,35,134141.

Van-den Driessche, P., Watmough, J.(2002). Reproduction numbers and subthreshold endemic equilibria for compartmental models of disease transmission. Math. Biosci, 180,29 48.

World Health Organization, Factsheet.http://www.who.int/mediac entre/factsheets/fs327/en/. (Accessed November, 2016).

Yakob,L., Clements, A.C.A.(2013). A mathematical model of Chikungunya dynamics and control: The major epidemic on Reunion Island. Journal of Plosone,8(3).e57448.dio:10.1371/journ al.pone. 0057448 . 